\title{
Development of Highly Sensitive Amperometric Biosensor for Glucose Using Carbon Nanosphere/Sodium Alginate Composite Matrix for Enzyme Immobilization
}

\author{
En HaN, ${ }^{\dagger}$ Xia LI, Jian-Rong CaI, ${ }^{\dagger}$ Hai-Ying CuI, and Xing-Ai Zhang \\ School of Food and Biological Engineering, Jiangsu University, Zhenjiang 212013, P. R. China
}

\begin{abstract}
In this study, we developed a highly sensitive amperometric biosensor for glucose detection based on glucose oxidase immobilized in a novel carbon nanosphere (CNS)/sodium alginate (SA) composite matrix. This hybrid material combined the advantages of CNS and natural biopolymer SA. This composite film was characterized by scanning electron microscope, electrochemical impedance spectroscopy and UV-vis, which indicated that the hybrid material was suitable for immobilization of glucose oxidase. Various experimental conditions were investigated that influenced the performance of the biosensor, such as $\mathrm{pH}$, applied potential and temperature. Under the optimum conditions, the biosensor showed excellent performance for glucose over a wide linear concentration range from $1.0 \times 10^{-6}$ to $4.6 \times 10^{-3} \mathrm{M}$ with a detection limit of $0.5 \mu \mathrm{M}$ based on a signal-to-noise ratio of 3. Furthermore, the biosensor exhibited excellent long-term stability and satisfactory reproducibility.
\end{abstract}

Keywords Carbon nanosphere, sodium alginate, hybrid material, amperometric biosensor

(Received June 24, 2014; Accepted July 14, 2014; Published September 10, 2014)

\section{Introduction}

Recently, amperometric biosensors based on enzyme catalysis have attracted considerable interest due to their potential applications in several areas such as clinical diagnostics, food safety, and environmental protection. ${ }^{1-4}$ In particular, the development of biosensors with rapidity of measurement and high sensitivity for glucose detection is important in biological and clinical fields, because glucose plays a crucial role in life processes and its concentration in blood is usually used as a clinical indicator of diabetes. ${ }^{5,6}$ However, the challenge existing in biosensor fabrication is to provide a suitable microenvironment for the immobilized enzymes on the electrode surface that present the full functionality of the biomolecules over a long time. Therefore, it is critical to choose suitable materials as enzyme immobilization matrixes for the design of glucose biosensors with high analytical performance and long-term stabilization.

The applications of nanomaterials, including gold nanoparticle, carbon nanotube (CNT), graphene and semiconductor quantum-dots to biosensor strategies hold great promise in realizing high analytical performance due to their versatile properties. ${ }^{7-10}$ Among these nanomaterials, carbon-based nanostructures have been widely used to immobilize enzymes due to their large surface area and fast electron transfer rate. ${ }^{11-13}$ Carbon nanosphere (CNS), a novel kind of carbon nanomaterial possessing homogeneity of particle size, good chemical stability, and porous nanostructure for large loading of guest molecules,

† To whom correspondence should be addressed.

E-mail: enhan@ujs.edu.cn (E. H.); jrcai@ujs.edu.cn (J.-R. C.) has been employed as an immobilizing material for the fabrication of biosensors. ${ }^{14-16}$ Moreover, the "green" synthesis under hydrothermal conditions can produce homogeneous CNS with surface-functional groups and good biocompatibility. ${ }^{17-19}$ However, the enzyme electrode constructed with pure CNS may suffer from serious problems when stored dry, such as a short lifetime of the biosensors due to the poor film-forming ability of pure CNS. Therefore, it is necessary to overcome the weakness of the pure CNS-modified biosensors and to improve their analytical performance.

Hydrogel material has been popularly used to develop biosensors such as hydrogel alginate, which is unbranched polysaccharide produced by marine brown algae..$^{20,21}$ Alginate displays several properties including biocompatibility, film-forming ability and gentle microenvironments to keep stability of the enzyme immobilized in the entrapped material. ${ }^{22}$ In this paper, we developed a composite matrix based on CNS and sodium alginate (SA) to design a highly sensitive glucose biosensor. Glucose oxidase was simply entrapped into this novel composite material, which could be readily immobilized onto the electrode surface and form a film after it was being dried (Scheme 1). The resulting biosensor based on the novel composite matrix combined the merits of CNS and SA, and exhibited excellent analytical performance for glucose, such as high sensitivity, good repeatability and long storage stability.

\section{Experimental}

\section{Reagents and materials}

Glucose oxidase (GOx) (EC1.1.3.4, from Aspergillus niger, $200 \mathrm{U} / \mathrm{mg}$ ) was purchased from Sigma-Aldrich Inc. (USA). 


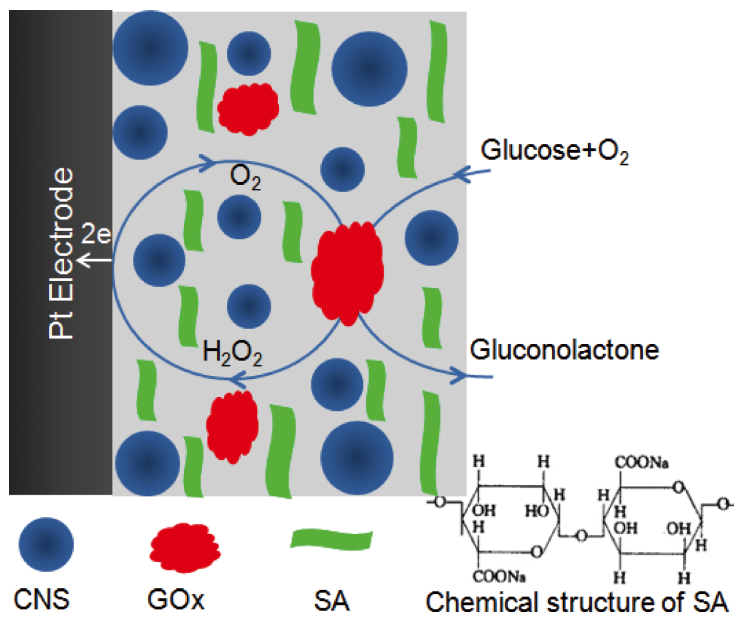

Scheme 1 Functioning scheme of CNS/SA/GOx modified electrode for glucose detection.

SA and glucose were obtained from Sinopharm Chemical Reagent Co., Ltd. (Shanghai, China). CNS was prepared with the glucose through the hydrothermal synthesis method. ${ }^{17}$ All other reagents were of analytical grade and used as received without further purification. All aqueous solutions were prepared in twice distilled water. Stock glucose solution was prepared daily and stored overnight to reach mutarotational equilibrium before use.

\section{Apparatus}

The UV-vis absorption spectra were recorded with a Cary-100 UV-vis spectrophotometer (Varian, USA). Electrochemical studies were carried out with CHI660d electrochemistry workstation (Shanghai CH Instrument Co., China). Micrographs were obtained with a JSM-7001F scanning electron microscope (SEM). The electrochemical measurements were performed with a conventional three-electrode system comprised of platinum wire as the auxiliary electrode, saturated calomel electrode (SCE) as the reference electrode, and modified platinum disk electrode as the working electrode. Electrochemical impedance spectroscopic (EIS) analysis was performed in $0.1 \mathrm{M}$ phosphate buffer solution (PBS) containing $5 \mathrm{mM} \mathrm{Fe}(\mathrm{CN})_{6}^{3-/ 4-}$ and $0.1 \mathrm{M} \mathrm{KCl}$ for a frequency range of $0.01 \mathrm{~Hz}-10 \mathrm{kHz}$.

\section{Preparation of the biosensor}

The platinum $(\mathrm{Pt})$ electrode was polished carefully using 0.3 and $0.05 \mu \mathrm{m}$ alumina slurry followed by rinsing thoroughly with distilled water. After successive sonication in 1:1 nitric acid, acetone and distilled water, the electrode was allowed to dry at room temperature. The SA solution $(0.2 \% \mathrm{w} / \mathrm{w})$ was prepared by dissolving SA in distilled water with the help of sonication. The CNS suspension $(2 \mathrm{mg} / \mathrm{mL})$ and GOx solution $(4 \mathrm{mg} / \mathrm{mL})$ were prepared by dispersing CNS and GOx in distilled water with stirring. Then, $10 \mu \mathrm{L}$ of the mixture solution with the optimal proportion (CNS/SA/GOx, w/w/w, 2:1:2) was spread on the surface of the Pt electrode, and then dried at $4^{\circ} \mathrm{C}$ in a refrigerator. CNS/GOx and GOx modified Pt electrodes via a cross-linked method by glutaraldehyde were used as controls.

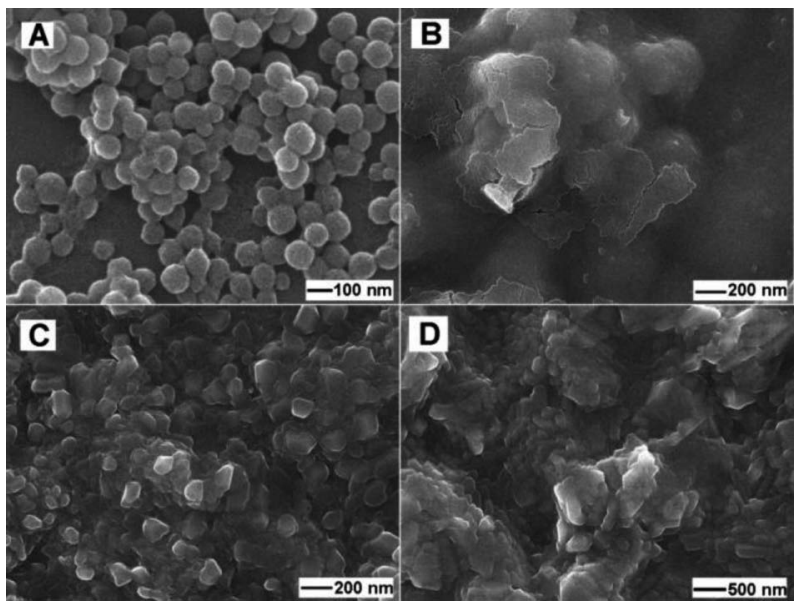

Fig. 1 SEM images of (A) CNS, (B) SA, (C) CNS/SA composite, and (D) CNS/SA/GOx composite films.

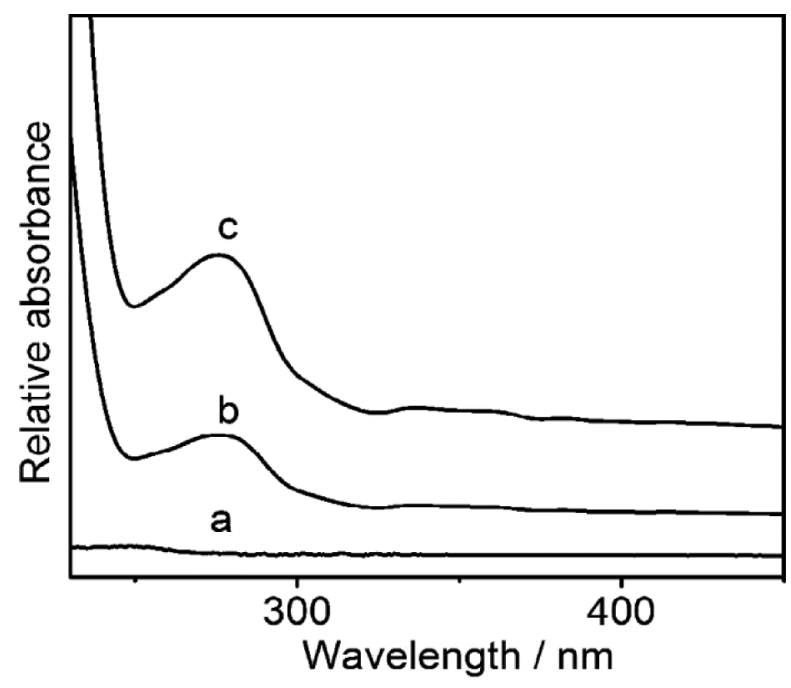

Fig. 2 UV-vis absorption spectra of (a) CNS/SA composite, (b) CNS/SA/GOx composite, and (c) GOx films.

\section{Results and Discussion}

Characterization of the CNS, SA,CNS/SA and CNS/SA/GOx films The surface morphologies of CNS, SA, CNS/SA and CNS/SA/GOx composite films were examined by SEM imaging. Figure 1A displays the typical morphology of the CNS, which exhibited uniform sphere particles with a diameter of approximately $100 \mathrm{~nm}$. The CNS film could be used as the matrix for loading biomolecules because of its large surface area, and good biocompatibility. However, the CNS film may present large pores for the loaded enzyme to leak into the solution by diffusing through it due to its poor film-forming ability. Figure 1B shows the morphology of a pure SA membrane, which displayed an excellent film-forming character. After mixing CNS and SA aqueous solution to form a homogenous solution, the composite could be readily immobilized onto the electrode surface and form a sturdy film (Fig. 1C). As shown in Fig. 1D, when GOx was entrapped in the CNS/SA film, more large particles were observed on the surface due to the aggregation of the enzyme molecules. This 
implied that the CNS/SA composite film was a suitable matrix for enzyme immobilization without the aid of other chemical cross-linking agents.

UV-vis spectroscopy was performed to study the possible

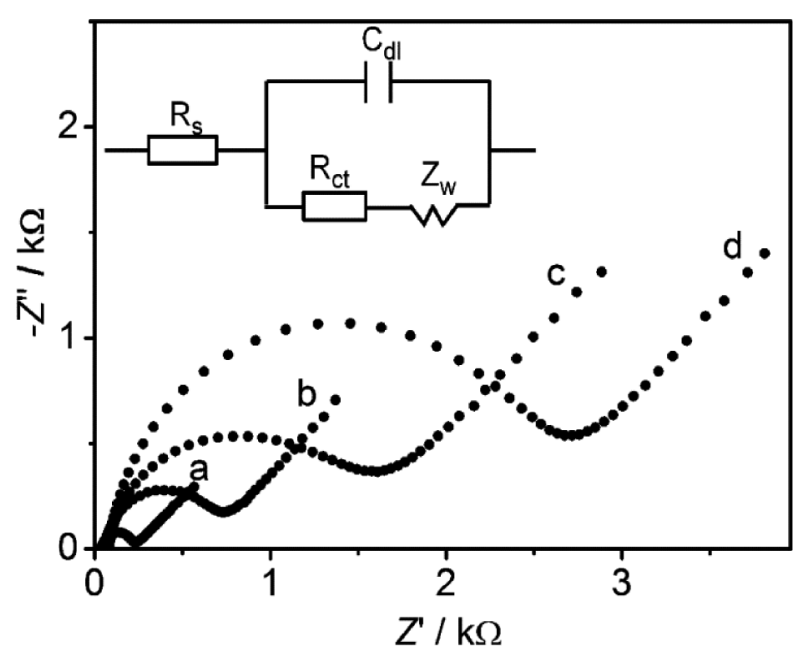

Fig. 3 Nyquist plots of EIS for (a) bare GCE, (b) CNS/GCE, (c) CNS/SA/GCE, and (d) CNS/SA/GOx/GCE in $0.1 \mathrm{M}$ PBS containing $0.1 \mathrm{M} \mathrm{KCl}$ and $10 \mathrm{mM}\left[\mathrm{Fe}(\mathrm{CN})_{6}\right]^{3-14}$ for a frequency range of $0.01-10 \mathrm{kHz}$. conformational change of GOx in the composite film. Figure 2 shows the UV-vis absorption spectra of CNS/SA (curve a), CNS/SA/GOx (curve b) and pure GOx (curve c) films on quartz slides. For the pure GOx, the absorption band at $276 \mathrm{~nm}$ was attributed to the characteristic of polypeptide chains in protein structure. ${ }^{23}$ The position of UV-vis absorption band for $\mathrm{CNS} / \mathrm{SA} / \mathrm{GOx}$ shifted by only $2 \mathrm{~nm}$ toward the red compared to that of pure GOx, suggesting that the GOx immobilized in CNS/SA composite film indeed maintained its native structure. ${ }^{24}$

The electron-transfer resistance $\left(R_{\mathrm{ct}}\right)$ at the electrode surface is an important parameter, representing the electron transfer kinetics of the redox probe at the electrode interface. Figure 3 displays a modified Randle's equivalent circuit and the Nyquist plots of the impedance spectroscopy of the different electrodes. The $R_{\mathrm{ct}}$ values for bare $\mathrm{Pt}, \mathrm{CNS} / \mathrm{Pt}, \mathrm{CNS} / \mathrm{SA} / \mathrm{Pt}$, and CNS/SA/ GOx/Pt electrodes were obtained as 224, 791, 1690 and $2863 \Omega$ (curves a-d) respectively. An obvious increase in the resistance was observed when GOx was embedded to the CNS/SA film (curve d). The increase in $R_{\mathrm{ct}}$ was caused by the hindrance of the macromolecular structure of GOx to the electron transfer.

\section{Optimization of experimental parameters}

The $\mathrm{pH}$ dependence of the biosensor over the $\mathrm{pH}$ range from 4.6 to 7.7 in PBS at $0.5 \mathrm{~V}$ in the presence of $0.5 \mathrm{mM}$ glucose was studied. As shown in Fig. 4A, the current signal firstly increased as the $\mathrm{pH}$ changed from 4.6 to 6.5 , and this was followed with a decrease in the $\mathrm{pH}$ range of 6.5 to 7.7. The


Modified electrodes

Fig. 4 Influence of experimental parameters of (A) $\mathrm{pH}$ of PBS at $0.5 \mathrm{~V}$, (B) applied potential in $\mathrm{pH}$ 6.5 PBS, and (C) temperature in $\mathrm{pH} 6.5 \mathrm{PBS}$ at $0.5 \mathrm{~V}$ on the biosensor response to $0.5 \mathrm{mM}$ glucose; (D) The responses of (a) GOx, (b) SA/GOx, (c) CNS/GOx and (d) CNS/SA/GOx modified electrodes to $0.5 \mathrm{mM}$ glucose in $0.1 \mathrm{M} \mathrm{pH} 6.5 \mathrm{PBS}$ at $0.5 \mathrm{~V}$. Insets in Fig 4B: Cyclic voltammogram $(0.3-0.65 \mathrm{~V})$ and current-time curve at $0.65 \mathrm{~V}$ of the biosensor to $0.5 \mathrm{mM}$ glucose. 
maximum current response was obtained at $\mathrm{pH} 6.5$, and this $\mathrm{pH}$ effect was similar to the optimal $\mathrm{pH}$ range of $5.0-6.0$ reported for the free GOx. ${ }^{25}$ Therefore, PBS at $\mathrm{pH} 6.5$ was selected as the electrolyte in subsequent experiments.

The influence of the applied potential for the glucose biosensor was also evaluated in the presence of $0.5 \mathrm{mM}$ glucose. The current could reach $95 \%$ of steady-state current within $8 \mathrm{~s}$ and kept stable for 2 min at $0.65 \mathrm{~V}$ (inset in Fig. 4B). As shown in Fig. 4B, the current response of the designed biosensor greatly increased with increasing applied potential from 0.30 to $0.50 \mathrm{~V}$, and then increased slightly in the applied potential range of 0.50 to $0.65 \mathrm{~V}$. The increasing current response with the applied potential could be attributed to the increased driving force for the electro-oxidation of enzymatically generated $\mathrm{H}_{2} \mathrm{O}_{2}$. However, the interference of easily oxidizable compounds could become stronger at a higher applied potential. Therefore, $0.50 \mathrm{~V}$ was selected as the optimal applied potential in subsequent experiments.

The effect of temperature on the biosensor response was investigated for $0.5 \mathrm{mM}$ glucose in the range of 5 to $55^{\circ} \mathrm{C}$. As shown in Fig. $4 \mathrm{C}$, the current signal increased greatly as the temperature changed from 5 to $45^{\circ} \mathrm{C}$, followed by a decrease in the temperature range of 45 to $55^{\circ} \mathrm{C}$. At higher temperatures, the current response decreased slowly because of the denaturation of the enzyme GOx. In the temperature range of 5 to $30^{\circ} \mathrm{C}$, the plot of $\ln i$ versus $T^{-1}$ showed a straight line $(r=0.991)$. According to the Arrhenius equation, the apparent activation energy $\left(E_{\mathrm{a}}\right)$ of the enzyme catalytic reaction was calculated to be $23.2 \mathrm{~kJ} \mathrm{~mol}^{-1}$ based on the slope of the straight

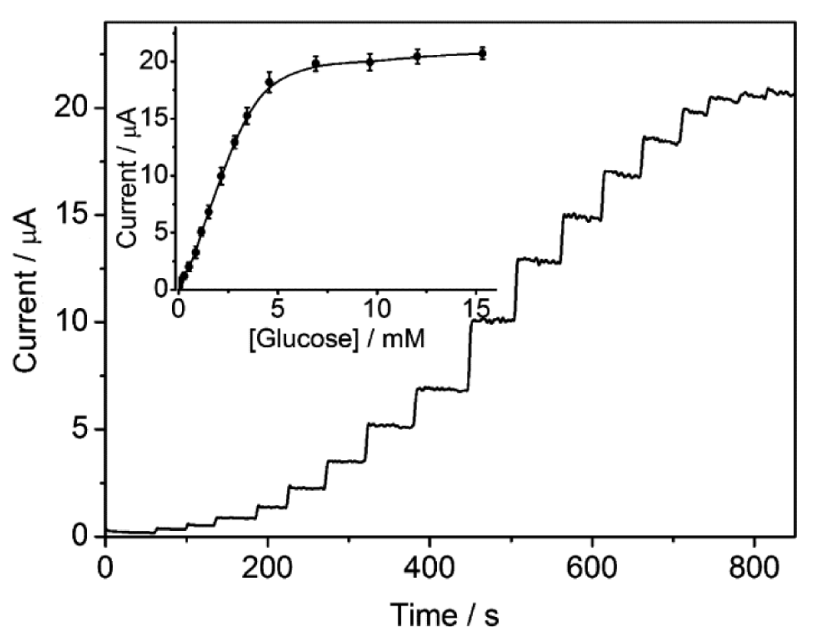

Fig. 5 Current-time response curve of the biosensor upon successive additions of glucose with different concentrations in $0.1 \mathrm{M}$ PBS ( $\mathrm{pH} 6.5$ ) at $0.5 \mathrm{~V}$. Inset: calibration curve of the designed biosensor for glucose detection. line. This value was similar to that of $22.1 \mathrm{~kJ} \mathrm{~mol}^{-1}$ for the layered double hydroxides/chitosan/GOx electrode. ${ }^{26}$ For a simple experimental procedure and long lifetime of the biosensor, further measurements were performed at $25^{\circ} \mathrm{C}$.

In order to compare the performance of different modified electrodes, the experiment further investigated the amperometric responses of GOx, SA/GOx, CNS/GOx and CNS/SA/GOx modified electrodes to $0.5 \mathrm{mM}$ glucose. As shown in Fig. 4D, compared with SA/GOx (column b) and CNS/GOx (column c) modified electrodes, the CNS/SA/GOx (column d) modified electrode displayed an obviously increasing current response. Significantly, the amperometric response of the biosensor based on CNS/SA nanocomposite was about twice higher than that of pure CNS modified electrode, proving the merits of the CNS/SA nanocomposite for biosensor construction.

\section{Amperometric biosensor detection of glucose}

Under the optimal conditions, the designed enzyme electrode exhibited a rapid response to the change of glucose concentration. Figure 5 shows a typical current-time plot for the amperometric biosensor on the successive additions of glucose to $10 \mathrm{~mL}$ PBS at an applied potential of $0.5 \mathrm{~V}$. The inset in Fig. 5 displays the corresponding calibration curve for the designed biosensor response to glucose. The linear range spanned the concentration of glucose from $1.0 \times 10^{-6}$ to $4.6 \times 10^{-3} \mathrm{M}$ with a correlation coefficient of 0.998 , and a low detection limit of $0.5 \mu \mathrm{M}$ at a signal-to-noise ratio of 3 . Its sensitivity was $63.8 \mathrm{~mA} \mathrm{M}^{-1} \mathrm{~cm}^{-2}$, which was much higher than that of glucose biosensor based on $\mathrm{ZrO}_{2} /$ chitosan/GOx $\left(22.3 \mathrm{~mA} \mathrm{M}^{-1} \mathrm{~cm}^{-2}\right),{ }^{27}$ Graphene/AuNPs/ GOx/Chitosan (17.5 $\left.\mathrm{mA} \mathrm{M}^{-1} \mathrm{~cm}^{-2}\right){ }^{28}$ and GOx/(AuNPs/ MWCNT $)^{5}\left(19.3 \mathrm{~mA} \mathrm{M}^{-1} \mathrm{~cm}^{-2}\right) .{ }^{29}$ This analytical performance of the proposed CNS/SA/GOx electrode was also compared with those reported in the literature for the GOx-based biosensors using other matrixes (Table 1). The high sensitivity and low detection limit of the CNS/SA/GOx sensor may be attributed to the combined merits of CNS and SA, such as good biocompatible and hydrophilic microenvironments, high film-forming ability and adsorption ability.

The apparent Michaelis-Menten constant, $K_{\mathrm{M}}^{\mathrm{app}}$, was calculated to be $11.2 \mathrm{mM}$ according to the Lineweaver-Burk equation. This value was smaller than the reported value for the free enzyme in solution $(27 \mathrm{mM}),{ }^{35}$ and the glucose biosensors based on nano- $\mathrm{CaCO}_{3} / \mathrm{GOx}$ electrode $(21.4 \mathrm{mM}) .{ }^{36}$ The smaller $K_{\mathrm{M}}^{\text {app }}$ value indicated that the GOx immobilized in the novel CNS/SA composite possessed high enzymatic activity and excellent affinity to glucose.

\section{Reproducibility and stability of the biosensor}

The reproducibility of the biosensor was estimated by determining $0.5 \mathrm{mM}$ glucose with six different electrodes constructed by the same procedure. The results revealed that the relative standard deviation (RSD) value for six electrodes

Table 1 Comparison with other reported biosensors for glucose detection

\begin{tabular}{|c|c|c|c|c|}
\hline Modified electeode & Sensitivity $/ \mathrm{mA} \mathrm{M}^{-1} \mathrm{~cm}^{-2}$ & Linear range/mM & Detection limit/ $\mu \mathrm{A}$ & Ref. \\
\hline Nafion/GOx/brushite & 52.5 & $0.009-3$ & 9 & 30 \\
\hline GOx/PdNPs/CS-GR & 31.2 & $0.001-1$ & 0.2 & 31 \\
\hline $\mathrm{MWCNT} / \mathrm{TiO}_{2} / \mathrm{HAP} / \mathrm{GOx}$ & 57.0 & $0.01-15.2$ & 2 & 32 \\
\hline GOx-Chi/pTBO/PB/rGO & 59.0 & $0.02-1.09$ & 8.4 & 33 \\
\hline GOx-pDA/GO & 28.4 & $0.001-4.7$ & 0.1 & 9 \\
\hline GOx/PtNPs/PAMAM-Sil-rGO & 24.6 & $0.01-8.1$ & 0.8 & 34 \\
\hline $\mathrm{CNS} / \mathrm{SA} / \mathrm{GOx}$ & 63.8 & $0.001-4.6$ & 0.5 & This work \\
\hline
\end{tabular}




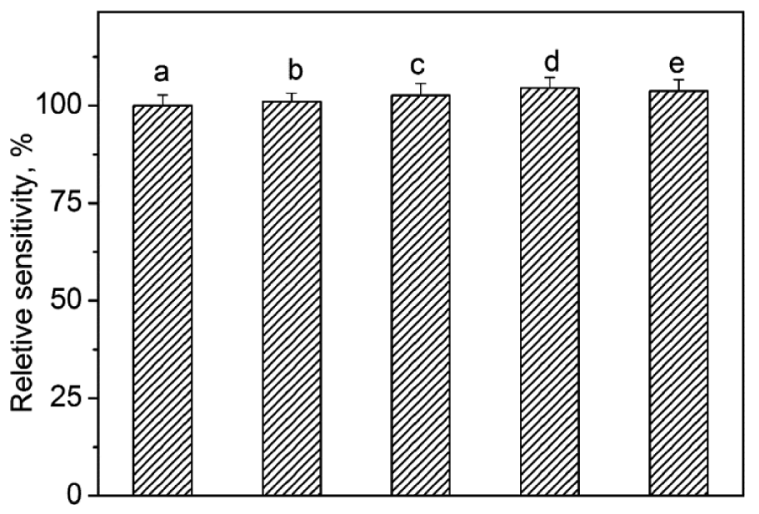

Fig. 6 Influence of interfering compounds on the response of CNS/SA/GOx electrode in $0.1 \mathrm{M} \mathrm{pH} 6.5 \mathrm{PBS}$ : (a) $1 \mathrm{mM}$ glucose, (b) $1 \mathrm{mM}$ glucose $+0.1 \mathrm{mM}$ L-cysteine, (c) $1 \mathrm{mM}$ glucose $+0.1 \mathrm{mM}$ ascorbic acid, (d) $1 \mathrm{mM}$ glucose $+0.1 \mathrm{mM}$ uric acid, and (e) $1 \mathrm{mM}$ glucose $+0.1 \mathrm{mM}$ p-acetamidophenol.

was $5.1 \%$, indicating good reproducibility of the fabrication protocol. When the CNS/SA/GOx electrode was stored at $4{ }^{\circ} \mathrm{C}$ and then measured at intervals over several days, no obvious decrease of the current response was observed for 28 days of storage. After 55 days the biosensor response still retained $83 \%$ of the initial response. This biosensor stability was much better than that of the CNS/GOx electrode for which the residual current response was only $62 \%$ after 28 days. The superior stability of the CNS/SA/GOx electrode was attributed to the good film-forming ability, the high mechanical strength and the biocompatible environment of the CNS/SA composite matrix.

\section{Interference and real sample analysis of the biosensor}

The influence of easily oxidizable interferents on the biosensor response to glucose was also examined. The interferences were estimated as a percentage of increase in the biosensor signal recorded in the presence of interferents, compared to the response to $1 \mathrm{mM}$ glucose alone (Fig. 6). The influence of L-Cysteine $(0.1 \mathrm{mM})$, ascorbic acid $(0.1 \mathrm{mM})$, uric acid $(0.1 \mathrm{mM})$, and $p$-acetaminophenol $(0.1 \mathrm{mM})$ to the glucose response were acceptable, namely $1.1-4.5 \%$. In order to demonstrate the practical usage of the biosensor, the $\mathrm{CNS} / \mathrm{SA} / \mathrm{GOx}$ electrode was further used to determine glucose concentration of human serum. The detection of glucose in serum was performed without any sample pretreatment except a dilution step. The glucose level for the serum sample was determined to be 4.06 which is close to the $4.17 \mathrm{mM}$ reading obtained by spectrophotometry, demonstrating good accuracy for detection of glucose in real samples.

\section{Conclusions}

In this work, a novel CNS/SA composite has been prepared for the immobilization of an enzyme to develop a glucose biosensor. This composite matrix combines the two advantages of CNS and SA, which include good biocompatibility, high film-forming ability and adsorption ability, and thus retains the native structure and bioactivity of the enzyme. The designed biosensor exhibits a variety of good analytical performances for glucose detection, including high sensitivity, wide linear range, good reproducibility and long-term storage stability. This CNS/SA composite provides a new approach and efficient matrix for the immobilization of enzymes and biomolecules, which will greatly facilitate the development of biosensors and other bioelectrochemical devices.

\section{Acknowledgements}

We gratefully acknowledge the financial support of the National Natural Science Foundation of China (Nos. 21205050 and 21205051), China Postdoctoral Science Foundation (No. 2012M511224) and Natural Science Foundation of Jiangsu University (No. 12JDG038).

\section{References}

1. A. W. Martinez, S. T. Phillips, and G. M. Whitesides, Anal. Chem., 2010, 82, 3.

2. C. N. Kotanen, F. G. Moussy, S. Carrara, and A. GuiseppiElie, Biosens. Bioelectron., 2012, 35, 14.

3. B. V. Dorst, K. Bekaert, E. R. Martin, W. D. Coen, P. Dubruel, R. Blust, and J. Robbens, Biosens. Bioelectron., 2010, 26, 1178.

4. P. Raghu, T. M. Reddy, K. Reddaiah, B. E. Swamy, and M. Sreedhar, Food Chem., 2014, 142, 188.

5. E. H. Yoo and S. Y. Lee, Sensors, 2010, 10, 4558.

6. M. Khan and S. Y. Park, Anal. Chem., 2014, 86, 1493.

7. Z. G. Gu, S. P. Yang, Z. J. Li, X. L. Sun, G. L. Wang, Y. J. Fang, and J. K. Liu, Electrochim. Acta, 2011, 56, 9162.

8. V. Mazeiko, A. K. Minkstimiene, A. Ramanaviciene, Z. Balevicius, and A. Ramanavicius, Sens. Actuators, B, 2013, 189, 187.

9. C. Q. Ruan, W. Shi, H. R. Jiang, Y. Sun, X. Liu, X. Y. Zhang, Z. Sun, L. F. Dai, and D. T. Ge, Sens. Actuators, B, 2013, 177, 826.

10. M. Wooten, S. Karra, M. G. Zhang, and W. Gorski, Anal. Chem., 2014, 86, 752.

11. E. Araque, C. B. Arenas, M. Gamella, J. Reviejo, R. Villalonga, and J. M. Pingarrón, J. Electroanal. Chem., 2014, 717, 96.

12. A. C. Torres, M. E. Ghica, and C. M. A. Brett, Anal. Bioanal. Chem., 2013, 405, 3813.

13. B. Unnikrishnan, S. Palanisamy, and S. M. Chen, Biosens. Bioelectron., 2013, 39, 70.

14. S. C. Tang, S. Vongehr, and X. K. Meng, J. Mater. Chem., 2010, 20, 5436.

15. D. Du, Z. X. Zou, Y. S. Shin, J. Wang, H. Wu, M. H. Engelhard, J. Liu, I. A. Aksay, and Y. H. Lin, Anal. Chem., 2010, 82, 2989.

16. J. R. Cai, L. N. Zhou, and E. Han, Anal. Sci., 2014, 30, 669.

17. X. M. Sun and Y. D. Li, Angew. Chem., 2004, 116, 607.

18. L. R. Kong, X. F. Lu, X. J. Bian, W. J. Zhang, and C. Wang, Langmuir, 2010, 26, 5985.

19. Q. N. Xu, F. Yan, J. P. Lei, C. Leng, and H. X. Ju, Chem. Eur. J., 2012, 18, 4994.

20. R. E. Ionescu, K. A. Rabeah, S. Cosnier, and R. S. Marks, Electrochem. Commun., 2005, 7, 1277.

21. B. Polyak, S. Geresh, and R. S. Marks, Biomacromolecules, 2004, $5,389$.

22. C. H. Liu, X. L. Guo, H. T. Cui, and R. Yuan, J. Mol. Catal. B: Enzym., 2009, 60, 151.

23. H.Y. Liu and N. F. Hu, Electroanalysis, 2007, 19, 884.

24. D. Shan, J. Zhang, H. G. Xue, S. N. Ding, and S. Cosnier, Biosens. Bioelectron., 2010, 25, 1427.

25. T. Godievargova, V. Konsulov, and A. Dimov, J. Membr. 
Sci., 1999, 152, 235.

26. Q. F. Shi, E. Han, D. Shan, W. J. Yao, and H. G. Xue, Bioprocess Biosyst. Eng., 2008, 31, 519.

27. Y. H. Yang, H. F. Yang, M. H. Yang, Y. L. Liu, G. L. Shen, and R. G. Yu, Anal. Chim. Acta, 2004, 525, 213.

28. C. Shan, H. Yang, D. Han, Q. Zhang, A. Ivaska, and L. Niu, Biosens. Bioelectron., 2010, 25, 1070.

29. P. Si, P. Kannan, L. Guo, H. Son, and D. H. Kim, Biosens. Bioelectron., 2011, 26, 3845.

30. M. S. P. Lopez and B. Lopez-Ruiz, Electroanalysis, 2011, 23, 280.

31. Q. Zeng, J. S. Cheng, X. F. Liu, H. T. Bai, and J. H. Jian,
Biosens. Bioelectron., 2011, 26, 3456.

32. J. Li, D. Kuang, Y. Feng, F. Zhang, and M. Liu, Microchim. Acta, 2012, 176, 73.

33. X. Bai, G. Chen, and K. K. Shiu, Electrochim. Acta, 2013, 89,454

34. E. Araque, C. B. Arenas, M. Gamella, J. Reviejo, R. Villalonga, and J. M. Pingarrón, J. Electroanal. Chem., 2014, 717, 96.

35. M. J. Rogers and K. G. Brandt, Biochemistry, 1971, 10, 4624.

36. D. Shan, M. J. Zhu, H. G. Xue, and S. Cosnier, Biosens. Bioelectron., 2007, 22, 1612. 